\title{
EL INVOLUCRAMIENTO DE LAS FAMILIAS EN LA EDUCACIÓN DE LOS NIÑOS. CUATRO REFLEXIONES PARA FORTALECER LA RELACIÓN ENTRE FAMILIAS Y ESCUELAS ${ }^{1}$
}

\begin{abstract}
The parent involvement in the education of the children. Four reflections to strengthen the relation between families and schools
\end{abstract}

http://dx.doi.org/10.22235/pe.v9i2.1298

\section{ALICIA RAZETO*}

Recibido: 06-06- 2016

Revisado: 18-07-2016

Aceptado: 20-07-2016

Resumen: Este ensayo aporta cuatro reflexiones para visibilizar la relevancia que tiene el involucramiento de las familias en el sistema educativo, como una variable de la calidad de la educación, y emprender políticas y programas que fortalezcan la relación entre las familias y las escuelas. Las reflexiones son: i) el actuar de las familias influye en el desempeño educativo de los niños; ii) las familias vulnerables están en desventaja desde el punto de vista de su capacidad para apoyar la educación de sus hijos y de relacionarse con la escuela; iii) el Estado valora la participación de las familias en la educación, aunque las iniciativas desplegadas

\footnotetext{
${ }^{1}$ Este artículo es un producto académico del Fondecyt de Iniciación № 11140679, 2014-2017, denominado "La visita domiciliaria como estrategia de intervención social para aumentar la participación de los padres en la educación de sus hijos en escuelas básicas municipales".

* Doctora en Ciencias de la Educación de la Pontificia Universidad Católica de Chile y Magister en Gestión y Políticas Públicas de la Universidad de Chile. Desde hace más de 12 años se desempeña como académica de la Escuela de Trabajo Social, Facultad de Ciencias Sociales de la Pontificia Universidad Católica de Chile.
} 
El involucramiento de las familias en la educación de los niños. Cuatro reflexiones para fortalecer la relación entre familias y escuelas.

son insuficientes y están desactualizadas; iv) no hay que partir de cero: estudios internacionales entregan recomendaciones para fortalecer la relación entre familias y escuelas.

Palabras clave: Relación escuela-padres, desempeño educativo, familia, educación

Abstract: This essay contributes four reflections to show the relevancy that has the involvement of the families in the educational system, understanding it as a variable of the quality of the education and, to undertaking policies and programs to strengthen the relation between the families and the schools. The worn out reflections consist of the following ones: i) to act of the families influences the success or educational failure of the children; ii) the vulnerable families are in disadvantage from the point of view of his aptitude to support the education of his children and of relating to the school; iii) the state values the participation of the families in the education, though the unfolded initiatives are insufficient and are out of date; iv) it is not necessary to depart from zero: international studies deliver recommendations to strengthen the relation between families and schools.

Key words: Relation school-parents, student performance, family, education

\section{INTRODUCCIÓN}

Podría plantearse que la familia tiene dos características propias: es una comunidad de pertenencia (Morandé, 1999), que forja identidad persona y en la que se desarrollan potentes vínculos socioafectivos entre sus miembros; y es una comunidad educativa (Santelices y Scagliotti, 2005) que forma a las personas a lo largo de toda la vida y potencia su desarrollo integral.

Otra de las características es su diversidad, puesto que la familia ha experimentado durante las últimas décadas importantes transformaciones sociales y culturales, entre las que se encuentran: la disminución de su tamaño y el aumento de los hogares unipersonales; la postergación del matrimonio y la maternidad; el aumento de la participación laboral de las mujeres; el aumento de la jefatura de hogar femenina y la consecuente sobrecarga de trabajo para la 
El involucramiento de las familias en la educación de los niños. Cuatro reflexiones para fortalecer la relación entre familias y escuelas.

mujer; y la diversidad de las estructuras familiares (Ministerio de Desarrollo Social, 2009). Pese a los cambios socioculturales que ha experimentado en las últimas décadas, la familia mantiene un cometido irreemplazable: educar a sus miembros para contribuir a su desarrollo a lo largo de toda la vida.

Según Scola (2012), la familia es un lugar educativo, "una comunidad de amor y de solidaridad insustituible para la enseñanza y transmisión de valores culturales, éticos, sociales, espirituales, esenciales para el desarrollo y bienestar de los propios miembros y de la sociedad" (p. 7). En la familia se forma la identidad de las personas, se satisfacen las necesidades básicas y de aprendizaje, se adquieren los hábitos respecto a la educación y trabajo, se aprende a convivir con otros, pues se socializan las normas, valores, el autocontrol, la responsabilidad, el desarrollo social, el equilibrio emocional y la autonomía (Morandé, 1999; Bolívar, 2006; Romagnoli y Gallardo, 2008; Aylwin y Solar, 2002). Vista así, la familia es la primera escuela de las virtudes sociales (Juan Pablo II, 1981) y es la célula básica de la cultura, de la transmisión de la sabiduría humana, que se cultiva y transmite de una generación a otra (Morandé, 1999).

Conscientes del valor del cometido educativo de la familia, es preciso reconocer que su ejercicio en la vida cotidiana enfrenta complejidades no menores. Entre ellas, determinar — con las nuevas configuraciones familiaresquién y cómo se hace cargo de dicha labor, en qué tiempos y con qué recursos y apoyo lo hace, en un contexto actual de difícil compatibilización entre el trabajo y la familia.

En esta tensión, la escuela se presenta como institución educativa formal de larga data, tradición y relevancia, que complementa la misión de la familia, al especializar y profundizar la educación del niño en un contexto colectivo. Podría decirse que la familia y la escuela se necesitan y, sin embargo, no siempre se buscan ni menos se encuentran.

Lo cierto es que entre familias y establecimientos educativos debería desarrollarse una relación colaborativa, una relación de sociedad o alianza (partnership) entre educadores, padres y otros actores de la comunidad, en la que 
El involucramiento de las familias en la educación de los niños. Cuatro reflexiones para fortalecer la relación entre familias y escuelas.

compartan la responsabilidad por el aprendizaje y el desarrollo, mediante un modelo de "superposición de las esferas de influencia entre la escuela, familia y comunidad para trabajar en conjunto con el propósito de guiar y apoyar el aprendizaje y desarrollo de los estudiantes" (Epstein, 2011, p. 43).

$\mathrm{Si}$ el complemento entre las familias y la escuela tiene su foco puesto en apoyar el aprendizaje y el desarrollo de los estudiantes, entonces el involucramiento de la familia en la educación debería desarrollarse tanto en los tiempos y espacios institucionales escolares -en las distintas actividades e iniciativas que la escuela gesta- como en los extraescolares o en los distintos momentos de la vida cotidiana familiar. Ambos tipos de involucramiento son indispensables para fomentar el aprendizaje integral y sostenible en el tiempo de los niños y jóvenes.

Si bien la familia es el primer y más importante lugar educativo, es preciso aceptar que tendencias y exigencias propias de la vida moderna -el individualismo, el trabajo exacerbado y deshumanizante, el consumo- han puesto bajo presión y en cuestión la capacidad de las familias para practicar su misión educativa. De esta manera, paulatinamente, la escuela ha tendido a asumir la responsabilidad de la educación de los niños y adolescentes, en razón de la falta de tiempo de los padres y su desconocimiento de cuán fundamental es su compromiso con la educación de sus hijos (Romagnoli y Gallardo, 2008).

Conjuntamente, las iniciativas y programas para fortalecer el rol educativo de la familia, en y desde la escuela, han sido insuficientes. Así lo afirman Weiss, Bouffard, Bridglall y Gordon (2009) quienes señalan que, a pesar de que durante más de 40 años se ha acumulado evidencia de que el involucramiento de la familia es uno de los predictores más potentes del éxito escolar de los niños, los recursos y compromisos para promover este involucramiento han sido pocos, débiles e inconsistentes.

Considerando estos desafíos, el presente ensayo aporta cuatro argumentos y reflexiones que se proponen visibilizar la relevancia que tiene el involucramiento de las familias en el sistema educativo, al comprenderlas como una variable de la 
El involucramiento de las familias en la educación de los niños. Cuatro reflexiones para fortalecer la relación entre familias y escuelas.

calidad de la educación (Valverde y Valdivia, 2007), y reforzar la importancia de emprender políticas y programas que fortalezcan la relación entre las familias y las escuelas. 
El involucramiento de las familias en la educación de los niños. Cuatro reflexiones para fortalecer la relación entre familias y escuelas.

\section{El actuar de las familias influye en el desempeño educativo de los niños}

Los padres asumen un papel significativo en el proceso de aprendizaje y socialización de los niños (Jadue, 2003), especialmente en la primera infancia. A partir de la segunda mitad del siglo XX, numerosos estudios confirmaron la influencia que pueden ejercer padres y madres en los resultados educativos de sus hijos (Hoover-Dempsey, Walker, Sandler, Whetsel, Green, Wilkins y Closson, 2006; Epstein y Van Voorhis, 2001; Henderson, Mapp y Jordan, 2002; Hill y Craft, 2003; Coleman, 1966; Rasbash, Leckie, Pillinger y Jenkins, 2010).

Uno de los primeros estudios en reconocer los efectos de la familia sobre el rendimiento académico fue realizado por James Coleman. En esa publicación se concluye que el factor más relacionado con el rendimiento de los estudiantes era la composición social del alumnado, vale decir, sus antecedentes y entorno familiar. Según Coleman, la familia no solo influye por su estatus económico, sino también por el apoyo fuerte y efectivo que puede brindar en la educación de los estudiantes (Coleman, 1966).

Esto lo confirma más tarde un estudio aplicado por Stevenson y Baker (1987) en una muestra representativa de hogares americanos, que arrojó lo siguiente:

- Las madres que más educación han recibido son las que más se involucran en la educación de sus hijos. Son quienes tienen más información sobre la escuela y las que más actúan para resolver los problemas de sus niños en el sistema escolar, en comparación con las madres menos educadas. Al mismo tiempo, son las madres que más se contactan con la escuela las que generan estrategias más amplias y complejas para apoyar la educación de sus hijos.

- El involucramiento de los padres en la educación de sus hijos está asociado positivamente con el desempeño escolar tanto de niños como de niñas. 
El involucramiento de las familias en la educación de los niños. Cuatro reflexiones para fortalecer la relación entre familias y escuelas.

- Los padres se involucran más en las actividades de los establecimientos educativos cuando sus hijos son pequeños:

Los padres parecen desconectarse de las actividades de la escuela una vez que sus hijos están en el carril correcto. $O$, parece ser que los padres se sienten más competentes ayudando a sus hijos más pequeños que a los mayores (Stevenson y Baker, 1987, p. 1356).

Estudios sobre eficacia escolar también han comprobado el valor que tiene la relación de las familias con las escuelas. Es el caso de los investigadores Deal y Peterson (2009), quienes concluyen que uno de los hallazgos más consistentes es que la participación de los padres provoca una diferencia significativa en el desempeño educativo de los estudiantes y también en la gestión del staff del establecimiento. En la misma línea, Harris (2009) establece que el involucramiento de los padres como aliados de las escuelas es una de las estrategias fundamentales de los modelos de mejoramiento escolar en establecimientos educativos vulnerables.

Por su parte, Murillo (2008) destaca a las familias de los estudiantes como uno de los actores que influyen en los desempeños educativos de la siguiente forma:

- La relación y apoyo que prestan al estudiante.

- La participación e involucramiento en la escuela.

- Las expectativas positivas que sostienen ante la escuela y profesores.

En Chile, un estudio realizado por UNICEF sobre las características de las escuelas efectivas encontró que todas realizaban acciones concretas, aunque diversas, para vincularse con las familias, apuntando a la construcción de una alianza entre ambas instituciones. Aquí dos aspectos se destacaban como comunes: que los padres manifestaban confianza en el establecimiento educativo 
El involucramiento de las familias en la educación de los niños. Cuatro reflexiones para fortalecer la relación entre familias y escuelas.

y las altas expectativas en el aprendizaje que podían conseguir sus hijos (UNICEF, 2004).

La incidencia de la familia en el desempeño educativo se ha investigado en los resultados de pruebas estandarizadas que miden calidad de la educación, como es el caso de la prueba PISA. Fernández y Del Valle (2013) concluyen que la brecha de rendimiento en la prueba PISA entre estudiantes de colegios públicos y privados costarricenses obedece, sobre todo, a las diferencias que existen en los factores familiares y personales de los estudiantes al momento de rendir la prueba. En el caso chileno, con la prueba SIMCE, los resultados oficiales han confirmado lo siguiente:

- Los estudiantes que tienen padres que los apoyan para que realicen sus tareas y trabajos, que conocen sus notas y los felicitan por sus logros, obtienen mejores resultados tanto en matemática como en lectura (SIMCE, Cuarto Básico, 2011).

- Se observan mejores resultados en comprensión de lectura en los estudiantes cuyos padres leen con ellos frecuentemente y más tempranamente (SIMCE, Segundo Básico, 2012).

- El involucramiento de los padres y apoderados se relaciona con mejores resultados de aprendizaje, y existen hasta 23 puntos de diferencia entre establecimientos de alto y bajo involucramiento (SIMCE, Segundo, Cuarto y Sexto Básico, 2013).

Toda la evidencia aquí referida reafirma que el actuar de las familias, y especialmente de los padres y madres, tiene un efecto valioso en el desempeño escolar de los niños, y es una variable significativa para alcanzar los propósitos del sistema educativo. Por tanto, el desafío de acercar a la escuela al logro de una buena calidad de la educación no solo depende de lo que sucede al interior del establecimiento, del desempeño de los profesores y del liderazgo del director, sino también de las familias, que están fuera del aula y cuyos hogares forman parte del 
El involucramiento de las familias en la educación de los niños. Cuatro reflexiones para fortalecer la relación entre familias y escuelas.

contexto escolar. En vista de ello, cobra sentido implementar estrategias más concretas y diferenciadas en las propias escuelas para incrementar la participación de las familias en la educación de sus niños.

\section{Las familias vulnerables están en desventaja desde el punto de vista de su capacidad para apoyar la educación de sus hijos y de relacionarse con la escuela}

Las capacidades de las familias para llevar a cabo su cometido educativo no están equitativamente distribuidas, puesto que la pobreza, el nivel educacional de los padres y su capital social influyen en el desarrollo y el desempeño escolar de los niños y adolescentes.

Estudios internacionales como los de Parcel, Dufur y Cornell (2010) coinciden en afirmar que la pobreza material es un factor de riesgo para los niños, ya que implica menor acceso a recursos educativos que apoyen el proceso de aprendizaje, como materiales y actividades educativas. Por su parte, Weiss et al (2009) establecen que los padres que viven en condiciones de pobreza o estrés económico experimentan más altos problemas de salud mental, que pueden limitar su habilidad para apoyar los estudios de los niños e incrementar la probabilidad de uso de prácticas punitivas. También enfrentan más barreras logísticas para acercarse a la escuela como falta de transporte, falta de flexibilidad de tiempo diario y falta de tiempo para vacaciones.

Algunas publicaciones chilenas también son consistentes con esta postura. Gubbins e Ibarra (2016) afirman que, a menores ingresos y capital cultural familiar, menor es la presencia de los padres en la escuela. De algún modo, las expectativas y actitudes de los padres hacia la educación varían según nivel socioeconómico e impactan en el interés académico de los niños.

Sin embargo, también se ha estudiado que el compromiso de los padres y la buena comunicación padres-hijos en temas escolares impactan en un resultado académico positivo (Gubbins e Ibarra, 2016). Por su parte, Jadue (2003) plantea que un estudiante que pertenece a una familia de nivel socioeconómico y cultural 
El involucramiento de las familias en la educación de los niños. Cuatro reflexiones para fortalecer la relación entre familias y escuelas.

bajo y uniparental "está en alto riesgo de presentar tanto problemas de rendimiento en la escuela como en sus vivencias personales y familiares" (Jadue, 2003, p. 120), debido a las características del medio en el que se desarrolla el niño: bajo nivel educativo de los padres y pobreza.

Como se ha mencionado, los padres de una familia en situación de pobreza suelen tener menor nivel educacional, factor que, según Parcel et al (2010), afecta el ambiente en el que crecen los niños, que a su vez tiene consecuencias en los resultados académicos. Según estos autores, la calificación educacional de la madre predice en su hijo la facilidad verbal, el logro en lectura y matemáticas. A su vez, un fuerte autoconcepto materno reduce el riesgo de problemas conductuales en los niños.

De la misma forma, una familia que está en situación de pobreza suele tener menor capital social (Parcel et al, 2010), lo que es una barrera si se considera que dichas conexiones sociales (con vecinos, personal de la escuela, compañeros de trabajo) podrían ampliar los recursos a los que los niños tendrán acceso.

Si a este conjunto de desventajas se le suma la histórica y persistente condición de alta segmentación social que presenta el sistema educacional chileno (UNICEF, 1999; Núñez, 2015), que agrupa a familias de estratos socioeconómicos similares, entonces el problema se agudiza. Así como la mixtura social dentro del aula contribuye al aprendizaje de los estudiantes (García-Huidobro, 2007), también podría decirse que la integración de familias que provienen de diversos estratos sociales y culturales produce efectos positivos de aprendizaje recíproco respecto de cómo apoyar la educación de sus hijos.

Un ambiente socialmente diverso fomenta el desarrollo del pensamiento crítico y permite aprender cosas nuevas, a interactuar con otros y a construirse redes sociales a medida que el círculo de personas, experiencias y contextos sociales se amplía (GarcíaHuidobro, 2007, p. 69). 
El involucramiento de las familias en la educación de los niños. Cuatro reflexiones para fortalecer la relación entre familias y escuelas.

De esta manera, no basta con valorar el rol de la familia ni desplegar estrategias para apoyar su involucramiento en la educación. También es necesario que estas iniciativas sean diferenciadas y especializadas según el contexto social de los estudiantes, ya que hay que considerar las barreras asociadas a la pobreza y, por tanto, planificar estrategias mucho más intensas, tanto en tiempo como en recursos necesarios para implementarlas. Así es como las redes que posea la organización escolar y su capacidad de conexión con otros servicios sociales de bienestar (Weiss et al. 2009) se presentan como estrategias valiosas para contextos socialmente vulnerables, siempre y cuando sean las escuelas y familias quienes asuman la responsabilidad del trabajo en alianza y no se delegue en otro tipo de organizaciones.

\section{El Estado valora la participación de las familias en la educación, aunque las iniciativas desplegadas son insuficientes y están desactualizadas}

Si bien las iniciativas son insuficientes y están desactualizadas, hay que reconocer algunos avances en el ámbito de la relación entre la familia y la escuela en Chile. Los principales son los siguientes: i) Política de participación de padres, madres y apoderados en el sistema educativo (Ministerio de Educación, 2002); ii) Reglamento que crea los Centros de Padres y Apoderados (Ministerio de Educación, 1990); iii) Marco para la Buena Enseñanza (Ministerio de Educación, 2008), que incluye como parte de las responsabilidades de los docentes las acciones de relación con las familias de los estudiantes. educativo

Política de participación de padres, madres y apoderados en el sistema En el año 2002, durante el gobierno de Ricardo Lagos Escobar, se publica por primera vez en Chile la Política de Participación de Padres, Madres y Apoderados en el Sistema Educativo. La ministra de Educación de la época, Mariana Aylwin, explica en su prólogo que la familia "es un factor insustituible en la formación 
El involucramiento de las familias en la educación de los niños. Cuatro reflexiones para fortalecer la relación entre familias y escuelas.

integral de las personas" (Ministerio de Educación, 2002, p. 2), y que por eso es preciso construir una alianza estratégica entre la familia y la escuela.

[El objetivo de la política es] generar condiciones institucionales y jurídicas que garanticen la promoción, fomento y desarrollo de la participación amplia, diversa y organizada de padres, madres y apoderados/as y de la comunidad en el sistema educativo. Esta participación se realiza en alianza con los demás actores de la comunidad educativa, con el fin de contribuir en la construcción, desarrollo y cumplimiento de su proyecto y metas educativo institucionales, en el marco de mejorar la calidad de la educación (Ministerio de Educación, 2002, p. 49).

Aun cuando la existencia de esta política sea significativa, ya han transcurrido casi 15 años desde su elaboración y no hay evaluaciones del proceso y resultados de su implementación. Probablemente, debido a que "no propone metas o resultados esperados que permitan realizar monitoreo o seguimiento de su implementación a nivel de los establecimientos educacionales, por lo que no resulta un instrumento útil para la dirección y gestión pedagógica de la escuela" (Gubbins, 2012, p. 71). Así es como, después de todo este tiempo, continúa vigente la afirmación de UNICEF, que planteaba que pese a la importancia que los padres tienen en el discurso de la reforma educacional, no se ha logrado una participación masiva, abierta y activa (UNICEF, 2002).

Si bien esta política visibiliza la relevancia que tiene la participación de la familia, no aporta un programa concreto de trabajo en las escuelas, probablemente porque no se gestó desde las escuelas, sino más bien desde los hacedores de política del Ministerio de la época. Además, la política no ha sido reformada ni actualizada, a pesar de los grandes cambios de contexto político, sociocultural y educacional que ha experimentado el país. 
El involucramiento de las familias en la educación de los niños. Cuatro reflexiones para fortalecer la relación entre familias y escuelas.

Reglamento General de Centro de Padres y Apoderados para los Establecimientos Educacionales reconocidos oficialmente por el Ministerio de Educación (Decreto 565)

En el año 1990 se crean los Centros de Padres y Apoderados, definidos en el artículo 1 como "organismos que comparten y colaboran en los propósitos educativos y sociales de los establecimientos educacionales de que forman parte".

[Los Centros] orientarán sus acciones con plena observancia de las atribuciones técnico-pedagógicas que competen exclusivamente al establecimiento [...] apoyarán organizadamente las labores educativas del establecimiento y estimularán el desarrollo y progreso del conjunto de la comunidad escolar (Ministerio de Educación, 1990, p. 2).

Según consta en el reglamento, la creación de los Centros de Padres obedece a la consideración de que:

- La familia constituye un agente esencial en la formación moral y ético social de los niños y jóvenes.

- Los fines de la educación presuponen, por una parte, el deber de estimular la convergencia de las influencias educativas de la escuela y la familia, y, por otra, mantener canales de comunicación que enriquezcan las relaciones entre ambas.

- La participación organizada de los padres y apoderados en la vida de la escuela hace posible integrar a los diversos estamentos de la comunidad escolar bajo similares y complementarios anhelos y propósitos educativos, además de materializar proyectos de colaboración mutua. 
El involucramiento de las familias en la educación de los niños. Cuatro reflexiones para fortalecer la relación entre familias y escuelas.

Desde 1990 al 2000 proliferaron los Centros de Padres, al punto de que en el año 2000 casi un $80 \%$ de los establecimientos declaraba tener uno (PNUD, 2000), lo que ascendía a un total de 8.096. A pesar de la masiva presencia de centros de padres en el país, estos adolecen de cierta fragilidad institucional, lo que está reflejado en que solo el $22 \%$ poseen personalidad jurídica (PNUD, 2000), condición que les permite una mayor autonomía financiera y reconocimiento formal, tanto de las autoridades del establecimiento como de las instituciones con las cuales se relacionan.

En este aspecto radica uno de los principales problemas del reglamento: la fragilidad del sistema de financiamiento de los centros. Al respecto, el reglamento establece que el principal financiamiento son las cuotas que sus miembros cancelan durante el año. Sin embargo, no se identifican otras fuentes de financiamiento posibles, que obliguen al Estado, municipio 0 al propio establecimiento. Es así como los recursos con los que cuenta esta organización dependerán exclusivamente de la capacidad y sistematicidad de pago de las familias, condición reproductora de inequidad social si se tiene en cuenta la homogeneidad de las familias de los estudiantes de las escuelas.

Pese a su relevancia, en más de 25 años este reglamento no ha experimentado cambios. Solo es preciso mencionar que el Decreto 24 del año 2005, que crea el Consejo Escolar, le asigna al presidente del Centro de Padres y Apoderados la responsabilidad de integrarlo².

\section{Marco para la Buena Enseñanza}

Como parte de la política de fortalecimiento de la profesión docente, el Marco para la Buena Enseñanza se crea en 2008 por parte del Ministerio de Educación

\footnotetext{
${ }^{2}$ El Consejo Escolar es un órgano principalmente informativo y consultivo, que tiene por propósito "propender a la activa participación de todos los actores de la comunidad escolar con el objeto de mejorar la calidad de la educación y los logros de aprendizaje en los establecimientos educacionales" (Ministerio de Educación, 2005, p. 1). A pesar de que la norma establece que es propositivo, no se especifican los aspectos en los que puede proponer. A su vez, este organismo podría llegar a ser resolutivo, siempre y cuando el sostenedor así lo decida.
} 
El involucramiento de las familias en la educación de los niños. Cuatro reflexiones para fortalecer la relación entre familias y escuelas.

(MINEDUC), como una guía para el desempeño profesional de los docentes del sistema escolar. En este documento se establece "lo que los docentes chilenos deben conocer, saber hacer y ponderar para determinar cuán bien lo hace cada uno en el aula y en la escuela" (Ministerio de Educación, 2008, p. 5).

Este marco establece varios dominios, uno de los cuales es el de Responsabilidades Profesionales. En este dominio se destaca la relevancia de que el profesor desarrolle relaciones de colaboración y respeto con los padres y apoderados, pues se considera fundamental involucrarlos en el mejoramiento de los aprendizajes y actitudes de los estudiantes. Así es como se recomienda que el profesor, de acuerdo a su cargo (profesor jefe, de ciclo o de asignatura), mantenga informados a todos los padres sobre los procesos de aprendizaje y los avances y dificultades de sus hijos. También, que cree relaciones de colaboración mutua con las familias y abra oportunidades para que los padres y apoderados puedan involucrarse en las actividades del establecimiento, aportando sus saberes, experiencias y sus deseos de colaboración.

Sin embargo, este Marco no especifica estrategias, indicadores o resultados esperados que orienten al profesor y al director sobre cómo llevar a cabo esta responsabilidad y cómo evaluarla (Gubbins, 2012), por lo que termina siendo una responsabilidad que queda a discreción de la decisión del profesor y/o del establecimiento.

\section{No hay que partir de cero: estudios internacionales entregan recomendaciones para fortalecer la relación entre familias y escuelas}

Los beneficios de la participación de las familias han sido bastante estudiados, aunque menos lo han sido las formas de incrementar la participación, es decir, las prácticas efectivas para aumentar el involucramiento de los padres con la escuela y con la educación de los hijos. También es preciso reconocer que, si bien la relación entre familias y escuela conduce a un terreno todavía novedoso y 
El involucramiento de las familias en la educación de los niños. Cuatro reflexiones para fortalecer la relación entre familias y escuelas.

complejo $^{3}$, hay estudios internacionales que marcan tendencia respecto a las orientaciones para incrementar la participación de los padres en la educación de los niños. Es propósito de esta cuarta reflexión esbozar las propuestas de investigadores reconocidos internacionalmente en esta temática.

Es interesante integrar la precisión conceptual que realizan Goodall y Montgomery (2014), quienes proponen un continuo entre el involucramiento y el compromiso parental, y apuntan a este último como el deseable, ya que envuelve un mayor compromiso o mayor sentido de identidad que el involucramiento. Ellos proponen tres tipos de relación en este continuo:

\section{Involucramiento parental con la escuela}

En este tipo de relación predomina la escuela en la relación con los padres, y es ella quien tiene el control en la entrega de la información. Los padres pueden participar en actividades, pero estas son creadas y controladas por la institución escolar. La mayoría de las actividades son realizadas en el establecimiento educativo o sus alrededores. Esta concepción es la que suele predominar, tanto en la política educacional chilena como en los discursos instalados en las escuelas respecto a la relación con las familias.

\section{Involucramiento parental con la educación}

Este nivel de participación puede tener lugar en la escuela o en el hogar y está caracterizado por un intercambio de información, entre los padres y el staff de la escuela, enfocada en el aprendizaje. La agencia de la relación de apoyo a los niños está compartida entre la escuela y los padres. Poco frecuente es encontrar este tipo de prácticas en los establecimientos educativos públicos chilenos, que suelen manifestar su distancia con las familias de los estudiantes debido a su poca presencia y activismo en la educación de los niños.

\footnotetext{
${ }^{3}$ En Chile no es habitual que, en su formación inicial, los profesores reciban formación en competencias o habilidades para el trabajo con las familias de los estudiantes.
} 
El involucramiento de las familias en la educación de los niños. Cuatro reflexiones para fortalecer la relación entre familias y escuelas.

Revista Páginas de Educación. Vol. 9, Núm. 2 (2016) ISSN: 1688-5287; e-ISSN: 1688-7468 
El involucramiento de las familias en la educación de los niños. Cuatro reflexiones para fortalecer la relación entre familias y escuelas.

Compromiso parental con el aprendizaje de los niños

En este nivel de relación el protagonismo lo tienen los padres, quienes toman decisiones de acción y participación. Estos están comprometidos con el aprendizaje de sus hijos, no debido a que está dictado por la escuela, sino porque está dentro de sus propias percepciones de su rol como padres.

Ahora bien, tanto Weiss et al (2009) como Epstein (2011) parten de la base de que tanto la escuela como las familias son responsables del aprendizaje y educación de los niños y adolescentes. Podría decirse que el involucramiento de las familias en la educación es una co-construcción, una responsabilidad compartida entre estas, las escuelas y las comunidades (Epstein, 2011; Weiss et al. 2009).

Según Epstein (1992) es importante que la institución escolar desarrolle prácticas para involucrar a las familias en la educación. Una de las primeras consideraciones que propone esta autora es que las prácticas deben ser:

1) Diferenciadas: de acuerdo a las distintas necesidades de las familias y de sus diversas formas de constitución. Hay que tener en cuenta la diferencia etaria de los estudiantes, sus niveles de madurez, los ciclos de vida de las familias y sus situaciones socioeconómicas. También es preciso diferenciar el contexto de los colegios y los niveles en los que los educadores llevan a cabo su trabajo educativo.

2) Iniciales: Las prácticas de involucramiento de las familias en la educación debieran partir y enfatizarse en el nivel preescolar y básico (Epstein, 1992). Esto, debido a que es en los primeros años cuando las familias y escuelas aprenden a respetarse y apoyarse mutuamente en las responsabilidades compartidas para la educación de los niños. Además, "la calidad de las alianzas tempranas establecen modelos y relaciones que pueden alentar o desalentar a los padres a continuar comunicándose con los profesores de sus niños en años posteriores" (Epstein 1992, p. 10). En esta etapa temprana, el principal propósito de la conexión entre escuela y 
El involucramiento de las familias en la educación de los niños. Cuatro reflexiones para fortalecer la relación entre familias y escuelas.

familia es establecer y fortalecer el desarrollo cognitivo, personal y social de los niños y prepararlos para el aprendizaje.

Estas dos características suponen que la escuela conoce a sus estudiantes y familias, y que es sensible a su diversidad. Además, exigen la presencia de un equipo profesional que gestiona el vínculo. Esto implica una capacidad y recursos que no siempre están instalados en la institución escolar y se convierten en una necesidad de primera línea para contar con iniciativas de esta naturaleza.

Tomando en cuenta lo anterior, algunas prácticas posibles de participación de las familias que contribuyan al aprendizaje de los niños (Epstein, 1992, pp. 1112) son:

- Parentalidad (parenting): se refiere a un tipo de práctica orientada a construir condiciones en el hogar que favorezcan el aprendizaje de los niños y su mejor comportamiento en la escuela. Es importante, para ello, ayudar a las familias a desarrollar conocimientos y habilidades para entender a los niños en cada edad y nivel de desarrollo. Esto puede realizarse a través de actividades tales como workshops en la escuela o en otros lugares; mensajes telefónicos computarizados; cursos o capacitación para padres; implementación de programas de apoyo familiar en áreas de salud, nutrición u otros; visitas domiciliarias; reuniones en el vecindario; entre otros.

- Comunicación (communicating): este tipo de práctica apunta a diseñar y conducir formas efectivas de comunicación bilateral entre escuelas y familias acerca de los programas escolares y el progreso de los niños. Esto se puede hacer a través de actividades tales como: conferencias con todos los padres al menos una vez al año; envío semanal o mensual a los hogares de una carpeta con el trabajo de los estudiantes para la revisión o comentarios de los padres; uso de un esquema regular de noticias, memos, llamados telefónicos, boletines, sitio web del centro y otro tipo de 
El involucramiento de las familias en la educación de los niños. Cuatro reflexiones para fortalecer la relación entre familias y escuelas.

comunicaciones; información a los padres sobre las políticas, programas 0 reformas de la escuela; información a los padres sobre la seguridad de Internet.

- Voluntariado (volunteering): se refiere a reclutar y organizar apoyo de los padres como voluntarios que pueden ayudar a los profesores, administradores o niños en clases o en otras áreas. Ejemplos de actividades de esta naturaleza son: voluntarios para la sala de clases 0 para la escuela en general; la disponibilidad de un centro de familia para el trabajo voluntario; reuniones y recursos para las familias; la implementación de una encuesta anual para identificar los talentos disponibles, tiempos y localización de los voluntarios; "patrullas" de padres u otras actividades que les den seguridad y operación a los programas de la escuela; cadenas telefónicas para proveer información a padres; entre otros.

- Aprendizaje en el hogar (learning at home): consiste en proveer información e ideas para las familias acerca de cómo ayudar a los estudiantes en el hogar con las tareas y actividades, decisiones y planificaciones relacionadas al currículo. Actividades que pueden implementarse bajo esta práctica son: otorgar información a las familias sobre los conocimientos y habilidades requeridas por los estudiantes en cada asignatura para cada año; información sobre la política de tareas para el hogar y cómo monitorear y discutir el trabajo escolar en el hogar; información sobre cómo apoyar a los estudiantes en mejorar sus habilidades en diversas clases y evaluaciones; establecer un esquema regular de tareas que requieran que el estudiante discuta e interactúe con sus familias respecto a lo que está aprendiendo en clases; calendario con actividades para padres y estudiantes para realizar en la casa o en la comunidad; actividades familiares de lectura, ciencia y matemáticas en la escuela; actividades de aprendizaje en vacaciones; entre otras.

- Participación en la toma de decisiones (decision making): se refiere a un tipo de práctica orientada a incluir a los padres en las decisiones de la 
El involucramiento de las familias en la educación de los niños. Cuatro reflexiones para fortalecer la relación entre familias y escuelas.

institución escolar, lo que favorece el desarrollo de padres líderes y representativos. Las actividades que pueden contemplarse son: activar organizaciones de padres, consejos o comités (por ejemplo, comité currículum, seguridad o personal); grupos de defensa independientes para negociar y trabajar para la reforma de la escuela y su mejoramiento; consejos o comités locales para el involucramiento de la familia y la comunidad; información de elecciones en el establecimiento educativo; redes para unir a todas las familias con los padres representantes.

- Colaboración con la comunidad (collaborating with the community): consiste en identificar e integrar recursos y servicios desde la comunidad para fortalecer los programas de la escuela, las prácticas de las familias y el aprendizaje de los estudiantes y su desarrollo. Las actividades posibles son: informar a estudiantes y familias de los programas y servicios de salud, culturales, recreacionales y de apoyo social disponibles en la comunidad; informar de actividades comunitarias que incrementen el aprendizaje de habilidades y talentos, que incluyen los programas de verano para estudiantes; otorgar servicios a la comunidad por los estudiantes y familias, a través del reciclaje, arte, música, teatro y otras actividades; entre otros.

\section{CONCLUSIONES}

A partir de la evidencia disponible sobre la responsabilidad que tiene la familia en la calidad de la educación de sus hijos, en este ensayo se han propuesto cuatro reflexiones para visibilizar la relevancia que tienen las familias en el sistema educativo y para sugerir la necesidad de instalar en la escuela nuevos programas y acciones que contribuyan a fortificar la relación con las familias.

En primer lugar, se mostró evidencia de estudios que demuestran que familias más involucradas con la escuela y más comprometidas en la educación tienen niños con un mejor desempeño que aquellas familias que no lo están. En segundo lugar, se compartieron antecedentes de estudios que establecen que las familias de bajos recursos tienen menos capacidad para relacionarse con la 
El involucramiento de las familias en la educación de los niños. Cuatro reflexiones para fortalecer la relación entre familias y escuelas.

escuela y apoyar la educación de sus hijos, cuestión que las estrategias de relación familia-escuela no deberían ignorar. En tercer lugar, se reflexionó críticamente en los avances y desafíos que tienen las políticas y programas en este ámbito de relación. En último lugar, se mostraron algunas orientaciones y estrategias concretas de participación familiar para el aprendizaje de los estudiantes.

Las reflexiones aquí presentadas comparten el convencimiento de que abordar dicha relación podría dar frutos positivos en la mejora de la calidad de los aprendizajes de los niños y adolescentes. Para hacerlo, es preciso iniciar el camino instalando capacidades en la institución escolar que refieran a la incorporación de un equipo profesional idóneo para promover la relación entre la escuela y las familias y, para ello, que sus líderes o quienes la administran sean sensibles y valoren este desafío.

En Chile se han incorporado a trabajar en los establecimientos educacionales profesionales del área psicosocial, como los psicólogos y trabajadores sociales, quienes asumen un importante rol en la relación con las familias de los estudiantes, especialmente los trabajadores sociales. Sin embargo, no están reguladas las condiciones de formación especializada que este tipo de profesionales debiera demostrar para trabajar en problemáticas sociales de alta complejidad, que a menudo se presentan en escuelas socialmente vulnerables. Tampoco existe una tipificación de los roles y funciones a realizar, ni menos una evaluación de la efectividad de sus prácticas.

Pero el desafío de involucrar a las familias en la educación de los niños no solo atañe al equipo psicosocial y al director, sino también a los profesores, quienes son los que mejor conocen las condiciones y posibilidades de los estudiantes e indirectamente sus contextos familiares. Solo con ellos será posible influir en la cultura escolar, a menudo cargada de prejuicios y desconfianza hacia las familias de los estudiantes, exacerbados en contextos socialmente vulnerables. En este aspecto, la formación docente es uno de los caminos más importantes y estratégicos para avanzar en estrechar la relación entre las escuelas y las familias, 
El involucramiento de las familias en la educación de los niños. Cuatro reflexiones para fortalecer la relación entre familias y escuelas.

tanto a nivel inicial como de formación continua. La comprensión de la diversidad familiar, la valorización del impacto que tiene la familia en los aprendizajes del niño y el manejo de estrategias efectivas de acercamiento e involucramiento son materias centrales a abordar en la formación universitaria de los profesores.

En este sentido, reconocer y valorar el potencial que cada familia representa, por más diversa que sea, se vuelve un paso necesario y fundamental para acercar la escuela a las familias. De alguna manera, lo que se requiere es que sea la propia escuela la que crea firmemente en la necesidad y en los efectos que tendrá la mayor presencia de las familias en la educación de los niños, de modo que no se terminen implementando obligadamente políticas o programas creados por otros.

\section{REFERENCIAS}

Aylwin, N. y Solar, M. (2002). Trabajo social familiar. Santiago: Ediciones Universidad Católica.

Bolívar, A. (2006) Familia y escuela: dos mundos llamados a trabajar en común, Revista de educación, 339, 119-146.

Coleman, J. (1966). Equality of educational opportunity study. Washington: Government.

Deal, T. y Peterson, K. (2009). Shaping School Culture: Pitfalls, Paradoxes, \& Promises. San Francisco: Josey-Bass.

Epstein, J. (1992). School and family partnerships. Report $n .{ }^{\circ}$ 3. Baltimore: Center on Families Communities, Schools and Children's Learning.

Epstein, J. y Van Voorhis, F. (2001). More than minutes: teachers' roles in designing homework. Educational Psychologist, 36 (3), 181-193.

Epstein, J. (2011). School, family and community partnerships. United States: Westview press.

Fernández, A. y Del Valle, R. (2013). Desigualdad educativa en Costa Rica: la brecha entre estudiantes de colegios públicos y privados. Análisis con los 
El involucramiento de las familias en la educación de los niños. Cuatro reflexiones para fortalecer la relación entre familias y escuelas.

resultados de la evaluación internacional Pisa. Revista de la Cepal, 111, 3757.

García-Huidobro, J. (2007). Desigualdad educativa y segmentación del sistema escolar. Revista Pensamiento Educativo, 40 (1), 65-85.

Goodall, J. y Montgomery, C. (2014). Parental involvement to parental engagement: a continuum. Educational Review, 66 (4), 399-410 http://dx.doi.org/10.1080/00131911.2013.781576

Gubbins, V. e Ibarra, S. (2016). Estrategias Educativas Familiares en Enseñanza Básica: Análisis Psicométrico de una Escala de Prácticas Parentales. Psykhe, 25 (1), 1-17. http://dx.doi.org/10.7764/psykhe.25.1.773

Gubbins, V. (2012). Familia y escuela: tensiones, reflexiones y propuestas. Reflexiones Pedagógicas, 44, 64-73.

Harris, A. (2009). Improving Schools in Challenging Contexts. Second International Handbook of Educational Change (pp. 693-706). Netherlands: Springer.

Henderson, A., Mapp, K. y Jordan, C. (2002). A new wave of evidence: the impact of school, family, and community connections on student achievement. Austin, TX: Southwest Educational Development Laboratory.

Hill, N. y Craft, S. (2003). Parent-school involvement and school performance: mediated pathways among socioeconomically comparable african american and euro-american families. Journal of Educational Psychology, 95 (1), 7483.

Hoover-Dempsey, K., et al. (2006). Why do parents become involved? Research findings and implications. The elementary school journal, 106 (2), 105-130.

Jadue, G. (2003). Transformaciones familiares en Chile: riesgo creciente para el desarrollo emocional, psicosocial y la educación de los hijos. Estudios Pedagógicos, 29, 115-126.

Juan Pablo II. (1981). Exhortación apostólica Familiaris Consortio, 22 de noviembre de 1981. Recuperado de: http://w2.vatican.va/content/john-paulii/es/apost exhortations/documents/hf ip-ii exh 19811122 familiarisconsortio.html 
El involucramiento de las familias en la educación de los niños. Cuatro reflexiones para fortalecer la relación entre familias y escuelas.

Ministerio de Desarrollo Social de Chile (2009). Resultados Familia Encuesta CASEN 2009. Recuperado de: http://observatorio.ministeriodesarrollosocial.gob.cl/layout/doc/casen/casen 2009 familia.pdf

Ministerio de Educación de Chile (1990). Modifica Decreto $n^{\circ} 565$, que aprueba reglamento general de centros de padres y apoderados para los establecimientos educacionales reconocidos oficialmente por el ministerio de educación. Recuperado de: http://www.leychile.cl/Navegar?idNorma=15819

Ministerio de Educación de Chile (2002). Política de participación de padres, madres y apoderados en el sistema educativo. Recuperado de: http://portales.mineduc.cl/usuarios/convivencia escolar/doc/2011030214163 40.Politica de Participacion de Padres Madres y Apoderados en el Sis tema Escolar.pdf

Ministerio de Educación de Chile (2005). Decreto 24 que Reglamenta Consejos Escolares. Recuperado de: https://www.leychile.cl/Navegar?idNorma=236237

Ministerio de Educación de Chile (2008). Marco para la buena enseñanza.

Recuperado de:

http://portales.mineduc.cl/usuarios/cpeip/File/Documentos\%202011/MBE20 08.pdf

Morandé, P. (1999). Familia y sociedad: reflexiones sociológicas. Santiago: Editorial Universitaria.

Murillo, J. (2008). Enfoque, situación y desafíos de la investigación sobre eficacia escolar en América. Eficacia escolar y factores asociados en América Latina y el Caribe. Santiago de Chile: UNESCO.

Núñez, I. (2015). Educación chilena en la República: Promesas de universalismo y realidades de inequidad en su historia. Psicoperspectivas. Individuo $y$ Sociedad, 14 (3), 5-16. 
El involucramiento de las familias en la educación de los niños. Cuatro reflexiones para fortalecer la relación entre familias y escuelas.

Parcel, T., Dufur, M. y Cornell, R. (2010). Capital at Home and at School: A Review and Synthesis. Journal of marriage and family, 72, 828-846. doi:10.1111/j.1741-3737.2010.00733.x

PNUD - Programa de Naciones Unidas para el Desarrollo (2000). Más sociedad para gobernar el futuro. Informe sobre Desarrollo Humano en Chile. Santiago de Chile: PNUD.

Rasbash, J., Leckie, G., Pillinger, R. y Jenkins, J. (2010). Children's educational progress: partitioning family, school and area effects. J. R. Statist. Soc. 173, 657-682.

Romagnoli, C. y Gallardo, G. (2008). Alianza Efectiva Familia Escuela: Para promover el desarrollo intelectual, emocional, social y ético de los estudiantes. Recuperado de: http://valoras.uc.cl/images/centrorecursos/familias/ValoresEticaYDesarrolloSocioemocional/Documentos/Alia nza-Efectiva-Familia-Escuela Para-promover.pdf

Santelices, L. y Scagliotti, J. (2005). El educador y los padres. Estrategias de intervención educativa. Santiago: Ediciones Universidad Católica de Chile.

Scola, A. (2012). Familia y sociedad. Revista Humanitas, 26, 5-16.

SIMCE (2011). Resultados Nacionales Simce. Recuperado de: http://www.agenciaeducacion.cl/biblioteca-digital/resultados-simce/

SIMCE (2012). Resultados Nacionales Simce. Recuperado de: http://www.agenciaeducacion.cl/biblioteca-digital/resultados-simce/

SIMCE (2013). Resultados Nacionales Simce. Recuperado de: http://www.agenciaeducacion.cl/biblioteca-digital/resultados-simce/

Stevenson, D., y Baker, D. (1987). The familyschool relation and the child's school performance. Child Development, 58, 1348-1357.

UNICEF (1999). Ciclo de Debates: Desafíos de la Política Educacional. Claves de la inequidad en la educación básica. Recuperado de: http://www.unicef.cl/archivos documento/42/inequidad.pdf 
El involucramiento de las familias en la educación de los niños. Cuatro reflexiones para fortalecer la relación entre familias y escuelas.

UNICEF (2002). Participación de los Centros de Padres en la Educación. Expectativas, demandas, desafíos y compromisos. Recuperado de: http://www.unicef.cl/web/wp-content/uploads/doc wp/Centro Padres1.pdf UNICEF (2004). ¿Quién dijo que no se puede? Escuelas Efectivas en Sectores de Pobreza. Recuperado de: www.unicef.cl/centrodoc/escuelas efectivas/escuela\%20efectivas.pdf

Valverde, P. y Valdivia, M. (2007). La participación de los padres en los jardines infantiles. Boletín de Investigación Educacional, 21 (2), 207-232.

Weiss, H., Bouffard, S., Bridglall, B. y Gordon, E. (2009). Reframing Family Involvement in Education: Supporting Families to Support Educational Equity. Recuperado de: http://www.equitycampaign.org/i/a/document/12018 equitymattersvol5 web. pdf 